
\title{
28 Research Square \\ One-pot Synthesis of Janus Nanosheets on Strengthening and Toughening Nanocomposite Double Network Hydrogels
}

\section{Shuning Chen}

Fujian Normal University

\section{Wei Qian}

Fujian Normal University

Jiawen Chen

Fujian Normal University

Jianrong Lin

Fujian Normal University

Qinhui Chen ( $\nabla$ chenqh@fjnu.edu.cn )

Fujian Normal University https://orcid.org/0000-0002-5377-4136

\section{Research}

Keywords: nanosheets, nanocomposite double network hydrogels, strengthen, toughen

Posted Date: March 3rd, 2020

DOl: https://doi.org/10.21203/rs.3.rs-15746/v1

License: (c) (i) This work is licensed under a Creative Commons Attribution 4.0 International License. Read Full License 


\section{Abstract}

Nanoparticles have leading strengthening and toughening effects on polymer matrix. Anisotropic Janus nanoparticles exhibit abundant interfacial behavior because their two surfaces possess different chemical properties. Herein, Janus nanosheets (JNSs) were synthesized by one-pot method. Different surficial morphology and chemical groups on each surface of JNSs were observed by SEM and AFM. In the process of tannic acid/polyacrylic acid (TA/PAA) polymerization, JNSs act as crosslinking agent to promote the formation of nanocomposite double network hydrogels. When the TA/PAA hydrogels were stretched, both strength and toughness of TA/PAA hydrogels are improved owing to the solid silica enhancement, the orientation-disorientation and the interfacial chemical bond of JNSs. Besides, adsorption-desorption of PAA molecular chains also toughens hydrogels. The tensile strength and the elongation at break of TA/PAA hydrogels containing $1.5 \mathrm{wt} \% \mathrm{JNSs}$ are $42 \mathrm{KPa}$ and $370.9 \%$, respectively. Compared with the TA/PAA without JNSs, they increase by $90.9 \%$ and $88.7 \%$. The recovery and selfhealing of hydrogels with JNSs were accelerated dramatically. Meanwhile, the anti-fatigue property was improved. The as-prepared anisotropic Janus particles synthesized by one-pot method on strengthening and toughening double network hydrogels provide a new idea for the synthesis of Janus nanosheets and the crosslinking of hydrogel.

\section{Introduction}

The nanoparticles have excellent strengthening and toughening effects. The high temperature resistance and the mechanical properties of polymer materials with nanoparticles can be improve effectively [1]. Janus nanoparticles comprise different components on the same surfaces [2-4]. Blends with Janus particles have remarkable properties and interesting physical and chemical phenomena, which have attracted increasing attention in the scientific community. At present, there are two main methods for preparing Janus nanosheets. The first one is to synthesize hollow spheres and then grind them [5]. The other is selective modification on isotropic nanosheets [6]. These methods are cumbersome and have a long preparation period.

Polymer hydrogels are polymers with high water content formed by physical or chemical crosslinking [7]. Because of softness, hydrogels are widely used in wearable devices, and have great potential in the field of replacement and repair of tissues [8,9]. Tissue replacement and repair require that hydrogels have extraordinary strength and toughness, so that they can be used with low friction, shock absorption, high toughness, high strength and other characteristics [10]. However, most synthetic and natural hydrogels are fragile.

People have been working hard to develop hydrogels with excellent toughness and strength. Double network gel (DN-gels) [11] and nanocomposite gel (NC-gels) [12] are two kinds of rigid hydrogels. DN hydrogel is a contrast structure composed of tightly cross-linked rigid polyelectrolyte first network and loosely crosslinked flexible neutral polymer second network [13]. Upon loading, the rigid PAMPs network breaks into fragments, dissipating energy by sacrificial bonds, while the flexible PAAm chains still has 
high stress and strain [14]. Because the first network is irreversibly broken, the hydrogel cannot continue to dissipate energy. Hydrogels exhibit similar properties to elastomers. This is the deficiency of DN gel $[15,16]$.

Hydrophilic monomers can form tough and elastic NC gels by in-situ polymerization in water containing clay nanosheets [17]. Due to strong adsorption, each clay plate can absorb multiple polymer chains, which is similar to physical crosslinking, forming a physical network [18]. Silica nanoparticles can strongly adsorb polymer chains and significantly improve the strength and toughness of hydrogels [1921]. When the polymer chain is stretched, it is separated from the clay surface that the strain is significantly increased [22]. The entropy elasticity of chains and the force between the polymer and clay will dissipate energy. After unloading, some desorbed chains are re-adsorbed on clay surfaces, and the elastic deformation of the polymer chain is restored [17, 23-25].

The combination of nanoparticles and double networks is an effective way to form rigid hydrogels [22, 25]. Since the two faces of Janus nanoparticles are similar to the two-phase properties of the blends, Janus particles can firmly "grasp" the two phases of the blends to improve the compatibility of two phases and mechanical properties of the blends [26]. Herein, Janus nanosheets (JNSs) have been synthesized by one-pot method. The method is facial to operate, high yield without further crushing. In this work, as-prepared JNSs were added to TA/PAA hydrogel to form double-network hydrogel nanocomposite. The effects of JNSs on the rheological properties, toughening, strengthening, anti-fatigue properties and accelerating self-healing of hydrogels were studied.

\section{Experimental Section \\ 2.1 Materials}

Tetraethyl orthosilicate (TEOS), ethanol, toluene, Span 80, hydrochloric acid ( $\mathrm{HCl}$ ), silane coupling agent (KH550), tannic acid (TA) and ammonium persulfate (APS) were purchased from Sinopharm Chemical Reagent Co., Ltd. Acrylic acid (AA), N, N'-methylene bisacrylamide (BIS), (3-Methacryloxypropyl) trimethoxysilane (KH570) and tetramethylethylenediamine (TMEDA) were purchased from Aladdin reagent. Deionized water $\left(\mathrm{H}_{2} \mathrm{O}\right)$ was distilled again before use. The stabilizer in TEOS was removed by vacuum distillation before using.

\subsection{Preparation of JNSs}

Aqueous phase is $10 \mathrm{~g}$ of $\mathrm{H}_{2} \mathrm{O}$ with a pH value of 2.5 adjusted by $2 \mathrm{M} \mathrm{HCl}$. The oil phase is a mixture of $5.2 \mathrm{~g}$ TEOS, $0.92 \mathrm{~g} \mathrm{KH} 550,1.2 \mathrm{~g} \mathrm{KH} 570,6 \mathrm{~g}$ Span 80 and $60 \mathrm{~g}$ toluene. The aqueous and oil phases were pre-emulsified for 15 minutes by mechanical stirring equipment and polymerized at $70{ }^{\circ} \mathrm{C}$ for 12 hours. The product was washed three times by ethanol and one time by water to remove the abundant solvent. As-prepared nanoparticles were obtained by a freeze drier. Scheme 1 shows the synthesis route of JNSs.

\subsection{Preparation of TA/PAA hydrogel}


The solution contained $8 \mathrm{~g}$ of $\mathrm{H}_{2} \mathrm{O}, 0.02 \mathrm{~g}$ of TA, $2.5 \mathrm{~g}$ of AA, $0.25 \mathrm{~g}$ of APS, $0.007 \mathrm{~g}$ of BIS, $40 \mu \mathrm{L}$ of TMEDA and a certain proportion $(0,0.5,1,1.5,2$ wt.\%, which signed as S0, S0.5, S1, S1.5 and S2 repectively) compared to AA of JNSs was mixed by ultrasonic for 30 minutes. Then, the solution was poured into the molds $(60 \mathrm{~mm} \times 10 \mathrm{~mm} \times 20 \mathrm{~mm}$ and diameter $25 \mathrm{~mm}$ ) and put into the oven to polymerize at $60{ }^{\circ} \mathrm{C}$ for 1 hour.

\subsection{Morphology characterization.}

The morphology of JNSs and the fracture surfaces of TA/PAA hydrogels were observed by a scanning electron microscope (SEM, JSM-7500F, JEOL, Japan) at an accelerating voltage of $5 \mathrm{kV}$. Before observation, all samples were vacuum-sputtered with platinum. The surface chemical elements composition of unmodified and modified JNSs was characterized by an EDX (Oxford energy-dispersive Xray) on the SEM at an accelerating voltage of $15 \mathrm{kV}$. The thickness of the sample was characterized by an atomic force microscope (AFM, Bruker MultiMode 8, Germany) in tapping mode (topography).

\subsection{Mechanical properties test}

Tensile tests of TA/PAA hydrogels are carried out by using a universal material test machine (Lloyd LR5KPlus, UK) by three test modes-pull to break test, loading-unloading cycle test and stress-relaxation test. In pull to break test, hydrogels are pulled at a tensile speed of $40 \mathrm{~mm} / \mathrm{min}$ at room temperature.

Three samples per hydrogel are performed in this manner, and the standard deviation of each test datum was determined. In the loading-unloading cycle test, the speed of tensile is kept at a constant of $40 \mathrm{~mm} / \mathrm{min}$ with 20 times, and the maximum strain is $200 \%$. In the stress-relaxation test, the hydrogel samples are quickly preloaded to different strains in $\approx 0.2-0.4 \mathrm{~s}$ and the stress-relaxation curves in $200 \mathrm{~s}$ are recorded.

\subsection{Self-Healing properties test}

Hydrogels were cut off from the middle and spliced together. The self-healing condition was observed in an oven at $60{ }^{\circ} \mathrm{C}$ for $2 \mathrm{~h}$. All self-healed samples are tested according to pull to break test.

\subsection{Rheology characterization}

Hydrogels were carefully transferred to the rheometer plate of the Dynamic Hybrid Rheometer (DHR-2, TA Instruments, USA) with a spatula prior to the measurement. The rheology experiments were then carried out using a frequency-sweep mode with frequency of $1-100 \mathrm{rad} \mathrm{s}^{-1}$ at $0.1 \%$ strain (geometry: Conical plate, angle: $5.805^{\circ}$, gap: $0.2 \mathrm{~mm}$, temperature: $25^{\circ} \mathrm{C}$ ).

\section{Results And Discussion \\ 3.1 Morphology of JNSs}

Figure 1(a) and 1(b) are SEM and AFM images of JNSs, respectively. According to the images captured by SEM, curved nanosheets with two different surfaces can be observed. The concave surface is rough 
whereas the convex surface is smooth. In order to characterize the chemical composition of JNSs, the element distribution on two faces of JNSs are examined by an attached EDX and relative results are showed in Fig. 1(d). The rough surface is rich of nitrogen element whereas the smooth surface is poor of nitrogen element. The different nitrogen element on both sides indicates that the chemical composition of the two sides of the nanosheets is different. The inner part of the hollow sphere is hydrophilic and the outer part is hydrophobic. The silane coupling agents of $\mathrm{KH}-550$ and $\mathrm{KH}-570$ align in a vertical direction to the interface of water-in-oil droplets, where the amino groups orient to aqueous phase and double bonds orient to oil phase. When all of siliceous precursors hydrolyze, the rough concave surface is covered with amino groups and the smooth convex surface is covered with double bonds. Thickness measurements (Fig. 1(c)) tested by AFM shows that the thickness of JNSs is about $6 \mathrm{~nm}$. The uneven baseline at both ends of the curve indicates that the JNSs are curved and the concave surface is upward.

The diagrammatic sketch of JNSs is shown in scheme 1. At room temperature under acidic conditions, the three silanes occur sol-gel process at the interface and forming micron core shell structure [5]. A water-in-toluene emulsion forms under the condition of oil soluble surfactant Span-80 existence. At room temperature, when the $\mathrm{pH}=2.5$, the sol-gel process takes place at the oil-water interface. The silane containing amino groups tend to be protonated with positive charge towards the internal water phase. The silane with double bond has better lipophilicity, facilitating to face the external oil phase. Two silanes are further crosslinked in long sol-gel process to form rigid silica microspheres. However, Span 80 occupies the partial space of the shell, which leads to an inhomogeneity of the silane shell. A temporary emulsion is formed until the stirring stopped, and the aqueous phase will rush out from weak point of the shell (spaces occupied by Span 80) because of osmotic pressure. Without the support of internal aqueous phase, the spherical shell will collapse and spontaneously rupture to form JNSs.

Figure 1. (a) SEM image of JNSs. (b) Topography image of JNSs tested by AFM. (c) Weight Percentage of Elements in JNSs surface. (d) Thickness measurement of JNSs.

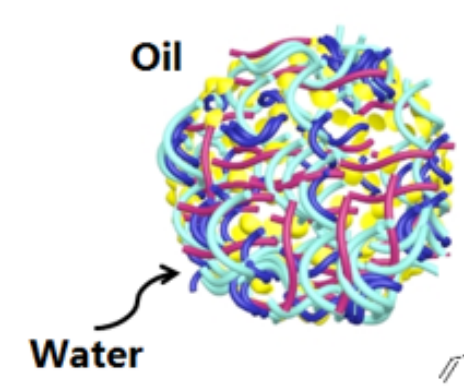

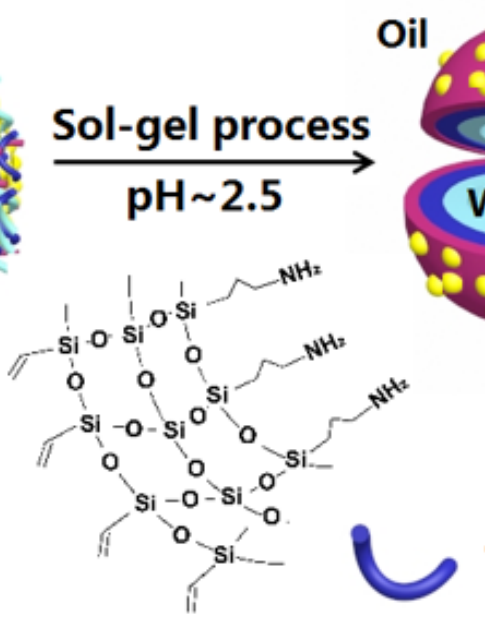

TEOS

$\stackrel{\text { Stop stirring }}{\longrightarrow}$

Fracture

Water

KH570

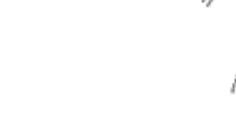

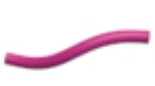




\subsection{Rheology characterization of TA/PAA hydrogels}

Figure 2. The rheological properties of the hydrogels
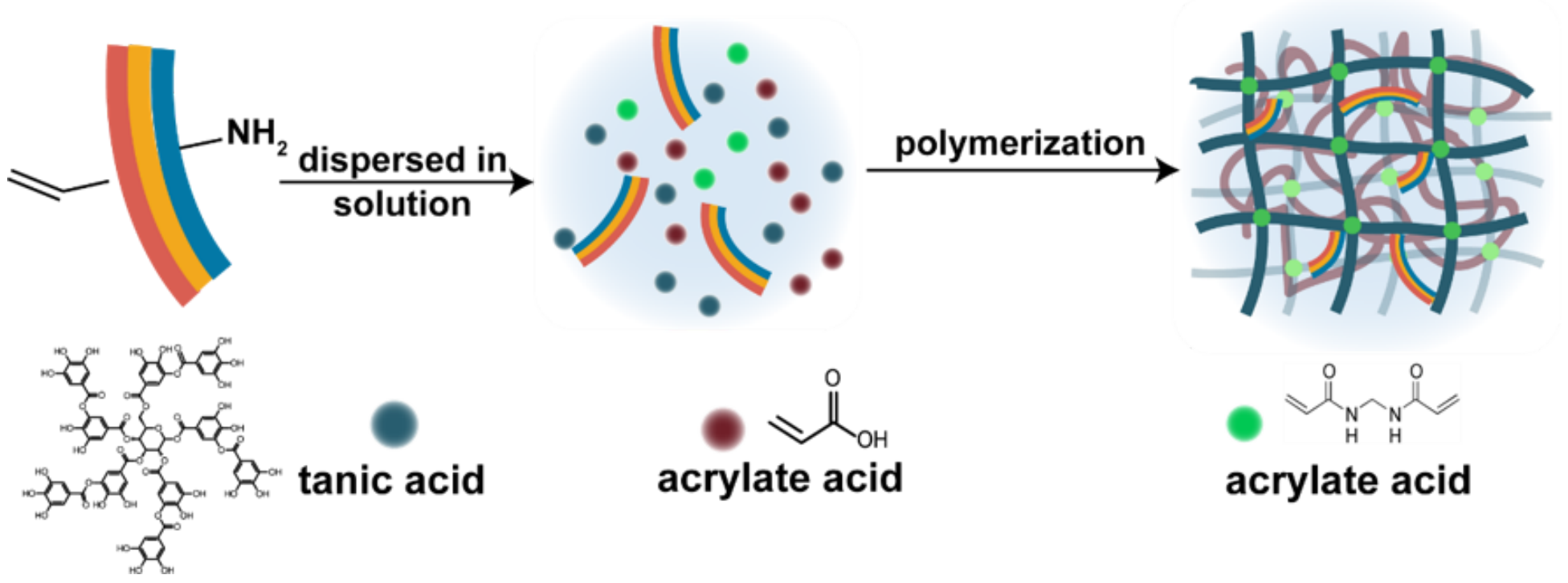

Scheme 2. JNSs were dispersed in solution and copolymerized at $60^{\circ} \mathrm{C}$ to prepare nanocomposite double- network hydrogels.

\subsection{Tensile properties of TA/PAA hydrogels}

The data of tensile strength of TA/PAA hydrogel are showed in Fig. 3. Tensile strengths of S0, S0.5, S1, $\mathrm{S} 1.5$ and $\mathrm{S} 2$ are $21 \mathrm{KPa}, 22 \mathrm{KPa}, 29 \mathrm{KPa}, 42 \mathrm{KPa}$ and $39 \mathrm{KPa}$, respectively. Elongations at break of the samples are $196.25 \%, 198.82 \%, 300.15 \%, 370.90 \%$ and $337.5 \%$, respectively. With the increase of JNSs content, the tensile strength and elongation at break of hydrogels increase. When content of JNSs reach $1.5 \mathrm{wt} \%$, the samples exhibit the most excellent comprehensive mechanical properties. The tensile strength of $\mathrm{S} 1.5$ increases by $90.9 \%$ and the elongation at break increases by $88.7 \%$ compared with that of S0. The further increase of JNSs leads to the decrease of fracture stress and the elongation at break (S2), which is due to the increase of stiffness of hydrogel networks with the JNSs content increases. However, these values are still higher than those of SO.

Figure 3. (a) Tensile strength and (b) stress-strain curves of TA/PAA hydrogels

\subsection{Cyclic tensile properties of TA/PAA hydrogels}

As a substitute for articular cartilage, hydrogels are required to undergo repeated stretching cycles so that they can complete sports and exercises. Full recovery of mechanical properties after loading is the prerequisite of cartilage. Therefore, the recovery rate of articular cartilage repair materials is an important index to evaluate the performance of articular cartilage repair materials. Figure 4 is the stress-strain curve of hydrogels obtained by loading-unloading experiment. Hysteresis loops occur after each loadingunloading process, which is a typical viscoelastic behavior. The hysteresis shows that there is an effective force dissipation mechanism in the hydrogel network, which can resist deformation. The main 
reasons for hysteresis loops are the relaxation effect of polymer chains and the loss of energy in the process of loading-unloading.

Figure 4. The stress-strain curves of hydrogels under loading and unloading.

The area surrounded by the loading curve and the X-axis represents the work done by the external force on the hydrogels $\left(S_{d}\right)$. The external force changes the conformation of the molecular segments and the arrangement of JNSs, which makes the molecular chains wrapped around JNSs to desorb, and provides the energy needed to overcome the internal friction between the segments when the segments move. The area surrounded by the unloading curve and $\mathrm{X}$-axis represents the work done by the hydrogels $\left(\mathrm{S}_{\mathrm{e}}\right)$. On the one hand, the elongated molecular chains and JNSs are restored to their original state, and the molecular chains are re-adsorbed and wound around JNSs. On the other hand, it is used to overcome the internal friction resistance between segments, that is, the energy released by hydrogels during unloading is converted into heat energy. The area of hysteresis loop $\left(S_{d}-S_{e}\right)$ is the energy absorbed minus the energy released, which indicates the energy loss of hydrogel during loading-unloading process. The larger the internal friction resistance, the more serious the lag phenomenon and the more the work consumed. The hysteresis rate $\left(\mathrm{S}_{\mathrm{d}}-\mathrm{S}_{\mathrm{e}}\right) / \mathrm{S}_{\mathrm{d}}$ represents the energy ratio of the loading process to the unloading process [28]. Table 1 summarizes the energy dissipation and cycles of hydrogels during loading-unloading test.

Table 1

The energy, hysteresis and cycle times of hydrogels in loading-unloading test.

\begin{tabular}{|llllll|}
\hline & $\mathbf{S}_{\mathrm{d}}$ (loading) & $\mathrm{S}_{\mathrm{e}}$ (unloading) & $\mathrm{S}_{\mathrm{d}}-\mathrm{S}_{\mathrm{e}}$ & $\left(\mathrm{S}_{\mathrm{d}}-\mathrm{S}_{\mathrm{e}}\right) / \mathrm{S}_{\mathrm{d}}$ & Cycle times \\
\hline S0 & - & - & - & - & 0 \\
\hline S0.5 & - & - & - & - & 1 \\
\hline S1 & 2512.03 & 2124.59 & 387.44 & 0.15 & $>20$ \\
\hline S1.5 & 3151.51 & 2561.01 & 590.50 & 0.19 & $>20$ \\
\hline S2 & 2854.07 & 2534.10 & 319.97 & 0.11 & $>20$ \\
\hline
\end{tabular}

The dissipated energy reflects the viscosity of the material and the degree of freedom of the molecular chain movement. Hydrogels with JNSs can recover quickly, especially S1.5. For the hydrogel without JNSs (S0), TA and PAA are only entangled because of the absence of a perfect dual network structure, and the cross-linking ordered structure has not yet been fully formed. When the content of JNSs reach a certain amount $(\mathrm{S} 1, \mathrm{~S} 1.5, \mathrm{~S} 2)$, the cycle times of hydrogels increase from one time to more than 20 times. This indicates that the effective energy dissipation can significantly improve the anti-fatigue property of hydrogels.

\subsection{The stress-relaxation properties of TA/PAA hydrogels}


One index of the mechanical properties of articular cartilage is stress relaxation. Cartilage can quickly damp mechanical loads, protect joints from mechanical damage, and can bear large mechanical loads steadily to avoid mechanical damage. Figure 5 shows the stress-relaxation of hydrogels. Different mechanical stresses were quickly applied to hydrogels (in $\approx 200 \mathrm{~ms}$ ) and the stress relaxation was observed. The hydrogels quickly relaxed the mechanical load by $\approx 50-70 \%$ and then remained stable without further mechanical softening. S0 was broken when a high stress of $1.78 \mathrm{MPa}$ was quickly applied. It was gratifying to see that with the induction of JNSs, the life span of the hydrogel greatly extended. This confirms that JNSs increases the crosslinking degree of hydrogels and reversible adsorption of PAA molecular chains is the main reason for stress relaxation. The relaxation time corresponds to the breakdown of TA network and desorption of PAA molecular chains. The loss of water in hydrogels also affected the relaxation time. The stress relaxation amplitudes became lower when the JNSs concentration in the hydrogel increased, while the relaxation times did not change obviously. This was because the amino and double bonds on JNSs reacted with the hydrogel. The hydrogel under the crosslinking of JNSs suffered little damage and possessed good recovery during rapid stretching.

Figure 5. The stress-relaxation test of hydrogels. The strain of each loading step increased by $30 \%$ in the range of $0 \%-150 \%$.

\subsection{Self-Healing Properties.}

Self-healing hydrogels can be easily reconstructed and reused even when part of the damage occurs. Therefore, it will greatly reduce the cost of maintenance and replacement of hydrogel devices. Figure 6(a) is a contrast photograph before and after hydrogel self-healing. After self-healing for 2 hours, there was an obvious gap in S0. Meanwhile, hydrogels with JNSs have healed well. JNSs provided a large amount amino group and double bond to react with the carboxyl group and double bond in PAA and TA. These bonds accelerated the self-healing of hydrogels. At the healing site of S0.5, color of the hydrogel is lighter compared with other parts of the sample. Tannic acid is easy to be oxidized to quinone under alkaline conditions, which is not conducive to oxidation reaction under acidic conditions, making the color lighter. The results showed that the color of healed So hydrogel was lighter due to the lack of amino group in JNSs. The pull to break test (Fig. 6(b)) was performed after self-healing. Compared with Fig. 3b, the strength and elongation of the gel decreased. S1.5 still maintained perfect tensile properties (Tensile strength was $0.031 \mathrm{MPa}$ and elongation at break was about $130 \%$ ), while the tensile strength of other gels decreased by more than half. These illustrated that self-healing speed of S1.5 was fast and its performance was good after self-healing owing to the reaction of JNSs and hydrogel.

Figure 6. Self-healing properties of hydrogels. (a) Photograph of hydrogel self-healing before and after treatment. (b) Tensile curves of hydrogel after self-healing.

\subsection{Possible strengthening and toughening mechanism}


To discover the enhancement mechanism of JNSs, the facture surfaces of five hydrogels were observed by SEM (Fig. 7). The cross section of SO is smooth. The addition of JNSs has little effect on the size and distribution of the aperture, but a more rugged cross section can be observed. Because of well dispersed three-dimensional JNSs, the amino and double bonds on each face of the JNSs can react with TA and PAA, which results stereoscopic interaction and generated multilayers pores. As a solid crosslinking agent, JNSs provides more crosslinking points, improves the crosslinking degree of hydrogels, and forms a double-network structure of stereo-interaction (S1.5). There are more paths to disperse stress, and cracks on wavy surfaces are shorter. This structure will effectively enhance the toughness and recovery of hydrogel. With the increase loading of JNSs, The tensile strength and elongation at break of S2 decreased due to the aggregated JNSs. The accumulated JNSs which were marked in red circles in Fig. 4(S2) could lead to defects in hydrogels and decrease the crosslinking density leading to deteriorated mechanical properties. The hydrogel transferred from soft material to rigid material by the introduction of excessive amount of JNSs, leading to a decrease of the elongation at break.

Figure 7. Morphology of fracture surface of TA/PAA hydrogels. The red circles in S2 mean aggregated JNSs.

From experimental data above, nanocomposite double network hydrogel has good energy dissipation and rapid recovery mechanism (scheme 3 ). The amino and double bonds on JNSs can react with PAA and TA, providing more crosslinking points and increasing the crosslinking degree of hydrogels (scheme 2). The rigidity of hydrogel increases with the increase of crosslinking degree, and the tensile strength of hydrogel increases with the increase of rigidity. Nanoparticles can not only induce the craze, but also prevent the crack from expanding. JNSs are anchored in hydrogels because of its physical or chemical bond with the matrix. Under the external force, the orientation of the JNSs along the direction of the force consumes a lot of energy, leading the tensile strength and elongation at break of the material to improve. When stretching, the TA chains of the first network are broken into fragments, and some of the chemical bonds between JNSs and the matrix break up. Energy consumption is sacrificial bonds in order to improve strengthen and toughness of hydrogels. The strong physical adsorption of polymer chains on the surface of silica nanoparticles is considered to be the reason for the super toughness of nanocomposite hydrogels. This is due to the energy dissipation caused by desorption of polymer chains from the surface of silica nanoparticles [29]. Under loading, the PAA chains adsorbed on JNSs will desorb and dissipate energy. When the external force is removed, the orientation of JNSs disorientate without external force. Because of the strong adsorption of JNSs, PAA chains are re-adsorbed on JNSs. Therefore, the hydrogel can recover quickly after the removal of external force and increase the times of cycle. 
(a)
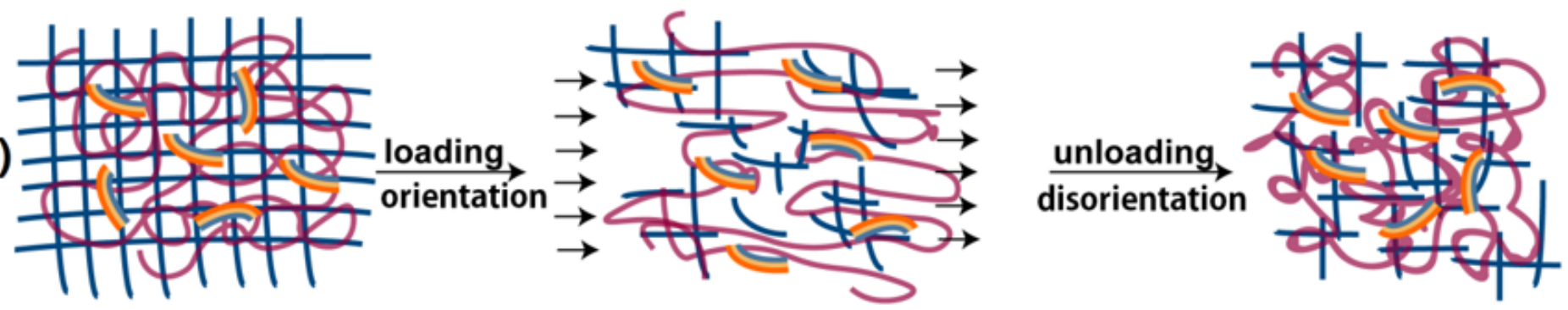

(b)
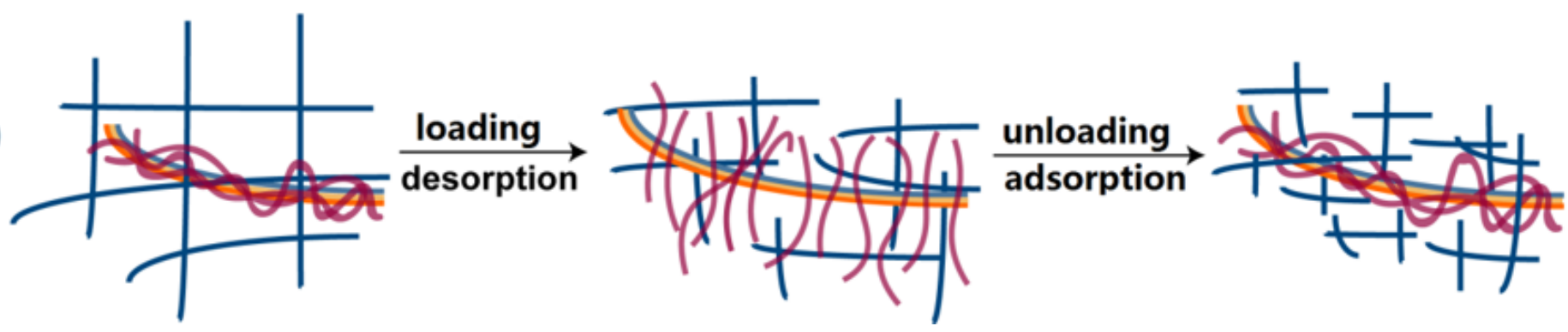

Scheme 3. Schematic illustration of the Strengthening and toughening mechanism of the JNSs-filled nanocomposite DN hydrogels

\section{Conclusion}

Janus nanoscale sheets were prepared by sol-gel method. Scanning electron microscopy (SEM) and atomic force microscopy (AFM) showed that the side of JNSs had amino group and double bond. Anisotropic Janus nanoparticles exhibit abundant interfacial behavior because their two surfaces possess different chemical properties.In the process of tannic acid/polyacrylic acid (TA/PAA)

polymerization, JNSs acted as crosslinking agent to form nanocomposite double-network hydrogel. When TA/PAA hydrogels were stretched, the strength and toughness of TA/PAA hydrogels were improved due to the enhancement, orientation-disorientation and surface chemical bonds of solid silica, as well as the adsorption-desorption of PAA molecular chains. The tensile strength and the elongation at break of TA/PAA hydrogels containing $1.5 \mathrm{wt} \%$ JNSs are $42 \mathrm{KPa}$ and $370.9 \%$, respectively. Compared with the TA/PAA without JNSs, they increase by $90.9 \%$ and $88.7 \%$. The recovery and self-healing of hydrogels with JNSs were accelerated dramatically, and the anti-fatigue property was improved. This work will provide new ideas for the synthesis, application of hydrogel contained of Janus materials.

\section{Declarations}

Ethics approval and consent to participate

No animals, human participants, human data, or human tissues were used in this study.

Consent for publication 
Not applicable.

Availability of data and materials

All data generated or analysed during this study are included in this published article.

Competing interests

There are no competing interests..

\section{Fundings}

This work was subsidized by the National Natural Science Foundation of China (No. 51773038) and the Natural Science Foundation of Fujian province (No. 2019J01669).

\section{Authors' Contributions}

Shuning Chen and Qinhui Chen performed the design of the experiments. Shuning Chen and Wei Qian were involved in the synthesis of JNSs and hydrogels. Shuning Chen and Jiawen Chen completed all the characterization and analyzed results. This manuscript was written by shuning Chen and Qinhui Chen. Jianrong Lin and Qinhui Chen contributed with crucial discussions and constructive reviews. All authors read and approved the final manuscript.

Acknowledgements

We thank Dr. Lixin Wu of Fujian Institute of Research on the Structure of Matter, Chinese Academy of Sciences for providing rheological testing equipment.

\section{References}

1. Li, H., X. Sui, and X.-M. Xie, High-strength and super-tough PA6/PS/PP/SEBS quaternary blends compatibilized by using a highly effective multi-phase compatibilizer: Toward efficient recycling of waste plastics. Polymer, 2017. 123: p. 240-246.

2. Guo, J., et al., Phase morphology evolution and compatibilization of immiscible polyamide 6/polystyrene blends using nano-montmorillonite. Polymer Engineering \& Science, 2018. 58(5): p. 752-758.

3. Bahrami, R., et al., Synergistic effects of Janus particles and triblock terpolymers on toughness of immiscible polymer blends. Polymer, 2017. 109: p. 229-237.

4. Nie, H., et al., Synthesis of Janus Rubber Hybrid Particles and Interfacial Behavior. Macromolecules, 2016. 49(6): p. 2238-2244.

5. Chen, Y., et al., Janus Nanosheets of Polymer-Inorganic Layered Composites. Macromolecules, 2012. 45(3): p. 1460-1467. 
6. de Leon, A.C., et al., Distinct Chemical and Physical Properties of Janus Nanosheets. ACS Nano, 2017. 11(7): p. 7485-7493.

7. Miyata, T., Preparation of smart soft materials using molecular complexes. Polymer Journal, 2010. 42(4): p. 277-289.

8. Webber, R.E. and C. Creton*, Large Strain Hysteresis and Mullins Effect of Tough Double-Network Hydrogels. Macromolecules, 2007. 40: p. 2919-2927.

9. Dhivya, S., et al., Nanohydroxyapatite-reinforced Chitosan Composite Hydrogel for Bone Tissue Repair in vitro and in vivo. Journal of Nanobiotechnology, 2015. 13: p. 40.

10. Lee, K.Y. and D.J. Mooney, Hydrogels for Tissue Engineering. Chemical Reviews, 2001. 101: p. 18691879.

11. Gong, J., Y. Katsuyama, and T.K.Y. Osada, Double-Network Hydrogels with Extremely High Mechanical Strength. Advanced Materials, 2003. 15: p. 1155-1158.

12. Haragushi, K. and T. Takehisa, Nanocomposite Hydrogels: A Unique Organic-Inorganic Network Structure with Extraordinary Mechanical, Optical, and Swelling/De-swelling Properties. Advanced Materials, 2002. 14: p. 1120-1124.

13. Nakajima, T., et al., Characterization of internal fracture process of double network hydrogels under uniaxial elongation. Soft Matter, 2013. 9(6): p. 1955-1966.

14. Gong, J.P., Why are double network hydrogels so tough? Soft Matter, 2010. 6(12): p. 2583.

15. Hu, J., et al., High Fracture Efficiency and Stress Concentration Phenomenon for Microgel-Reinforced Hydrogels Based on Double-Network Principle. Macromolecules, 2012. 45(23): p. 9445-9451.

16. Na, Y.-H., et al., Necking Phenomenon of Double-Network Gels. Macromolecules, 2006. 39: p. 46414645.

17. Haraguchi, K. and H.-J. Li, Mechanical Properties and Structure of Polymer-Clay Nanocomposite Gels with High Clay Content. Macromolecules, 2006. 39;: p. 1898-1905.

18. Haraguchi, K., Nanocomposite hydrogels. Current Opinion in Solid State and Materials Science, 2007. 11(3-4): p. 47-54.

19. Lin, W.-C., et al., Large Strain and Fracture Properties of Poly(dimethylacrylamide)/Silica Hybrid Hydrogels. Macromolecules, 2010. 43(5): p. 2554-2563.

20. Yang, J., et al., Studies on the properties and formation mechanism of flexible nanocomposite hydrogels from cellulose nanocrystals and poly(acrylic acid). Journal of Materials Chemistry, 2012. 22(42): p. 22467.

21. Yang, J., et al., Synthetic and viscoelastic behaviors of silicananoparticle reinforced poly(acrylamide) core-shell nanocomposite hydrogels. Soft Matter, 2013. 9(4): p. 1220-1230.

22. Cong, H.P., P. Wang, and S.H. Yu, Highly elastic and superstretchable graphene oxide/polyacrylamide hydrogels. Small, 2014. 10(3): p. 448-53.

23. Gaharwar, A.K., et al., Transparent, elastomeric and tough hydrogels from poly(ethylene glycol) and silicate nanoparticles. Acta Biomater, 2011. 7(12): p. 4139-48. 
24. Haraguchi, K., T. Takehisa, and S. Fan, Effects of Clay Content on the Properties of Nanocomposite Hydrogels Composed of Poly(N-isopropylacrylamide) and Clay. Macromolecules, 2002. 35: p. $10162-$ 10171.

25. Lian, C., et al., Self-Reinforcement of PNIPAm-Laponite Nanocomposite Gels Investigated by Atom Force Microscopy Nanoindentation. Macromolecules, 2012. 45(17): p. 7220-7227.

26. Xu, W., et al., Study on the Compatibilizing Effect of Janus Particles on Liquid Isoprene Rubber/Epoxy Resin Composite Materials. Industrial \& Engineering Chemistry Research, 2017. 56(47): p. 1406014068.

27. Han, L., et al., Mussel-Inspired Adhesive and Tough Hydrogel Based on Nanoclay Confined Dopamine Polymerization. ACS Nano, 2017. 11(3): p. 2561-2574.

28. Hu, J., et al., Microgel-Reinforced Hydrogel Films with High Mechanical Strength and Their Visible Mesoscale Fracture Structure. Macromolecules, 2011. 44(19): p. 7775-7781.

29. Okay, O. and W. Oppermann, Polyacrylamide-Clay Nanocomposite Hydrogels: Rheological and Light Scattering Characterization. Macromolecules, 2007. 40: p. 3378-3387.

\section{Figures}



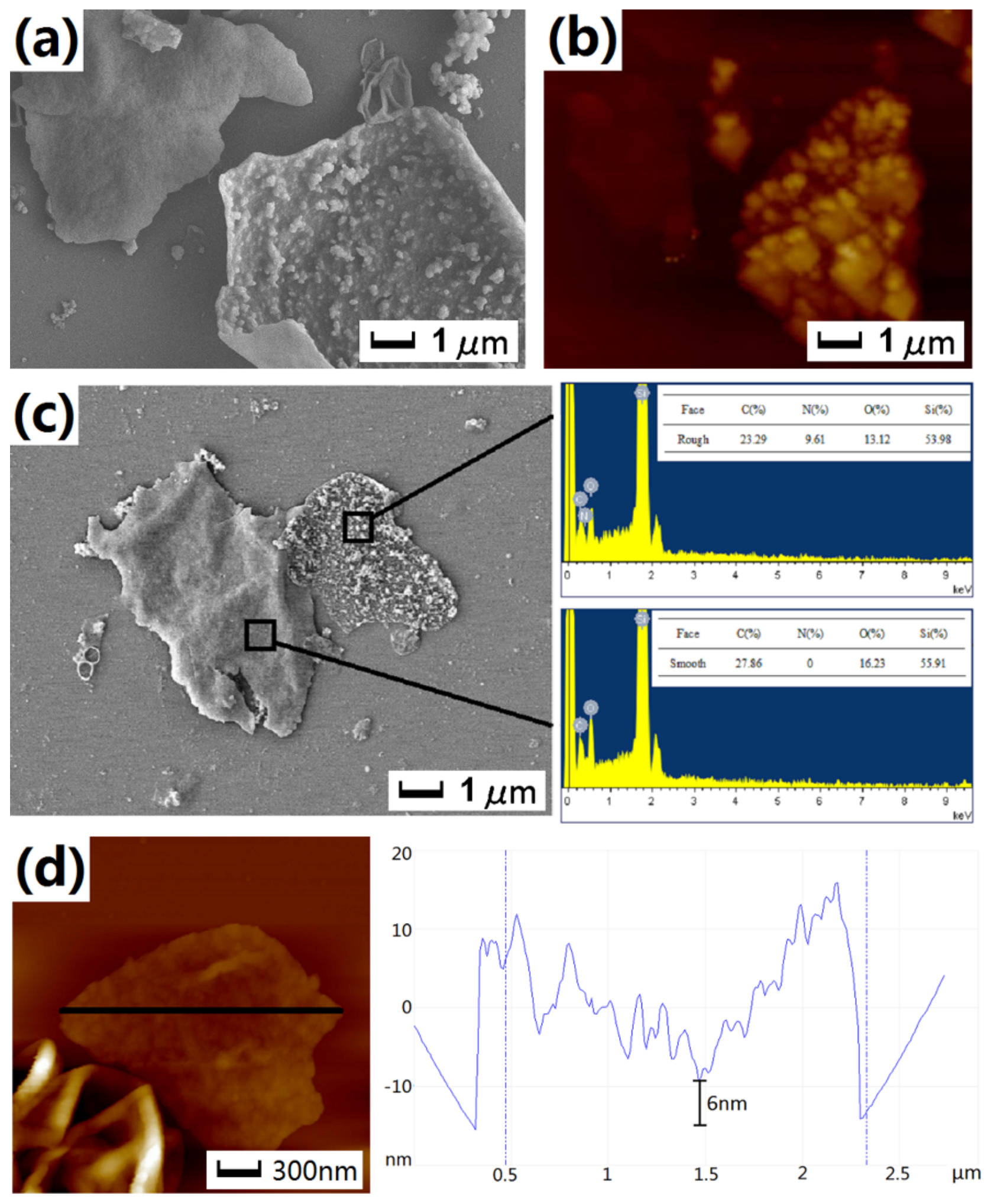

Figure 1

(a) SEM image of JNSs. (b) Topography image of JNSs tested by AFM. (c) Weight Percentage of Elements in JNSs surface. (d) Thickness measurement of JNSs. 

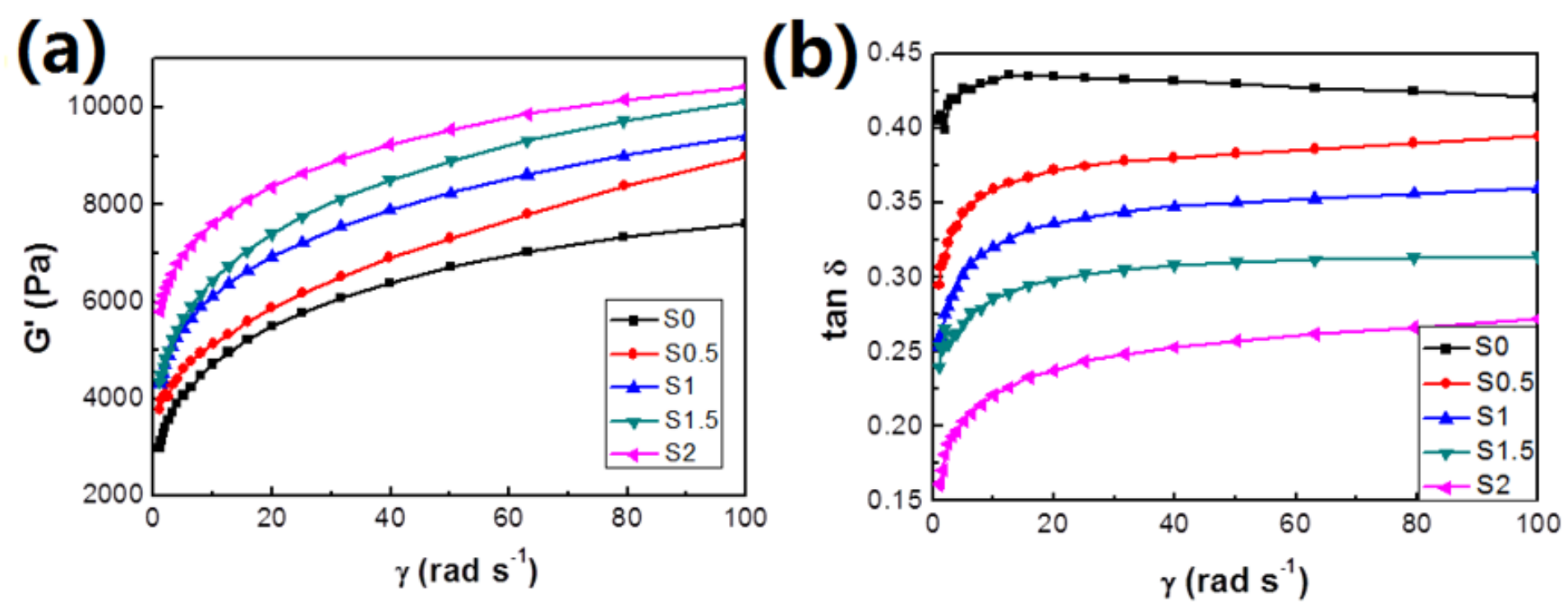

Figure 3

The rheological properties of the hydrogels
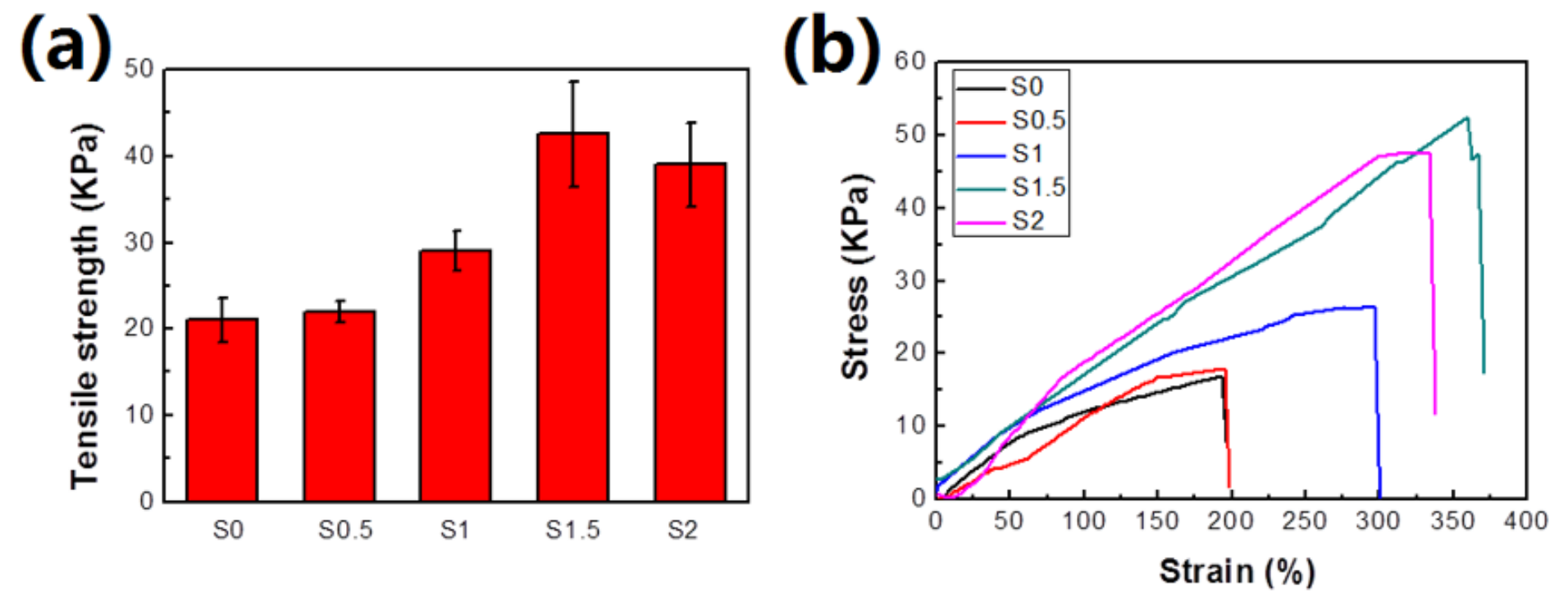

Figure 5

(a) Tensile strength and (b) stress-strain curves of TA/PAA hydrogels 


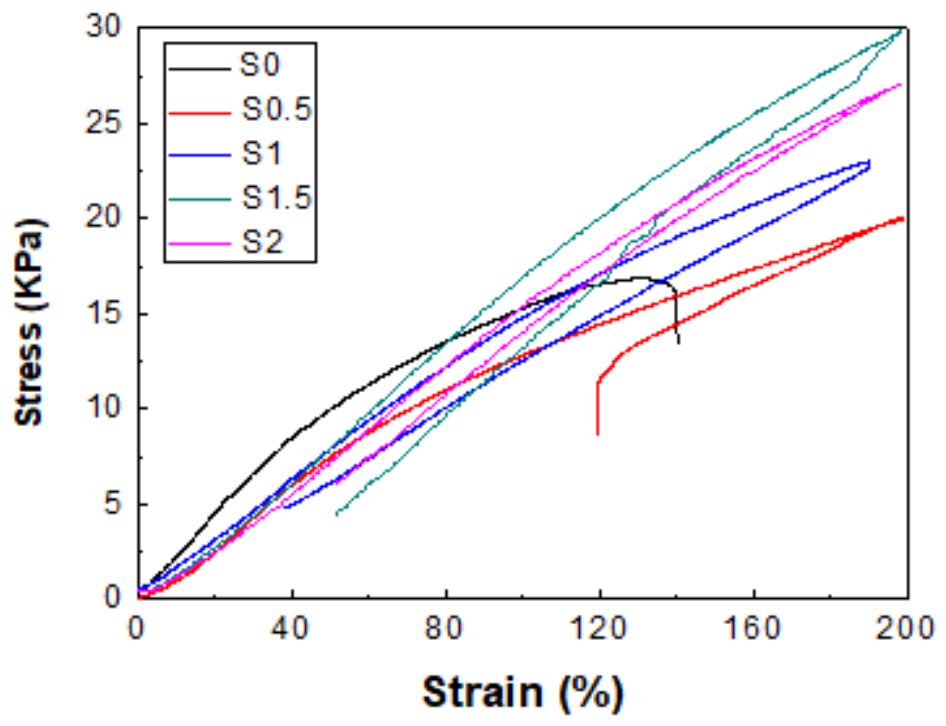

Figure 7

The stress-strain curves of hydrogels under loading and unloading.

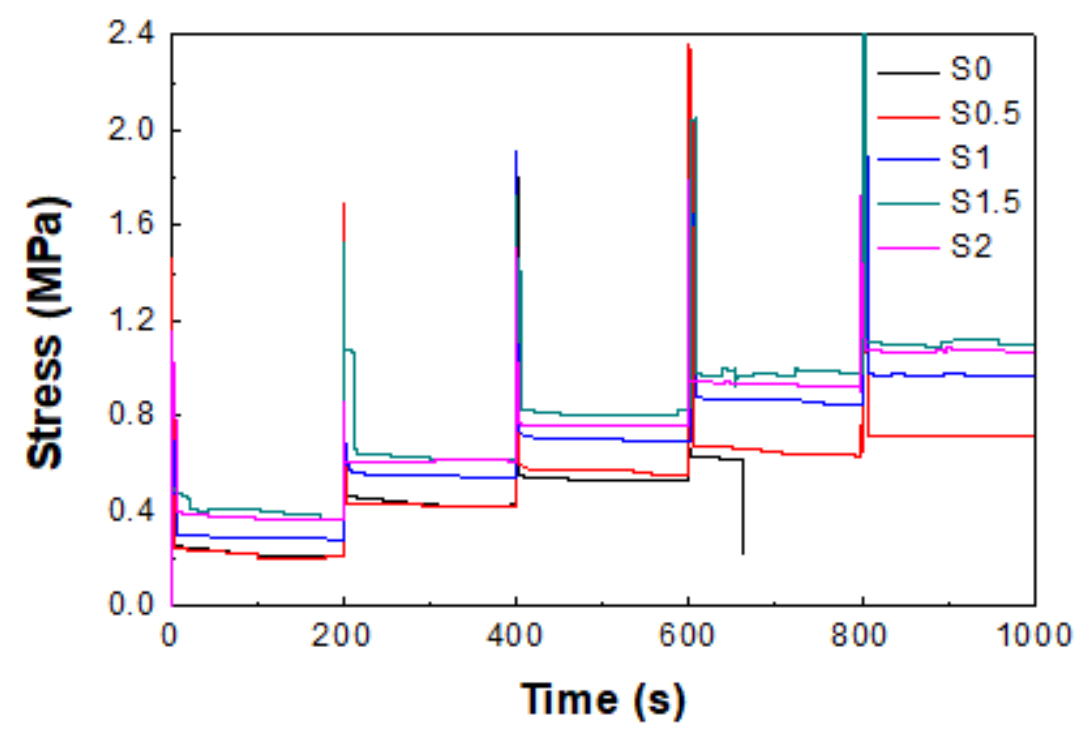

Figure 9

The stress-relaxation test of hydrogels. The strain of each loading step increased by $30 \%$ in the range of $0 \%-150 \%$. 

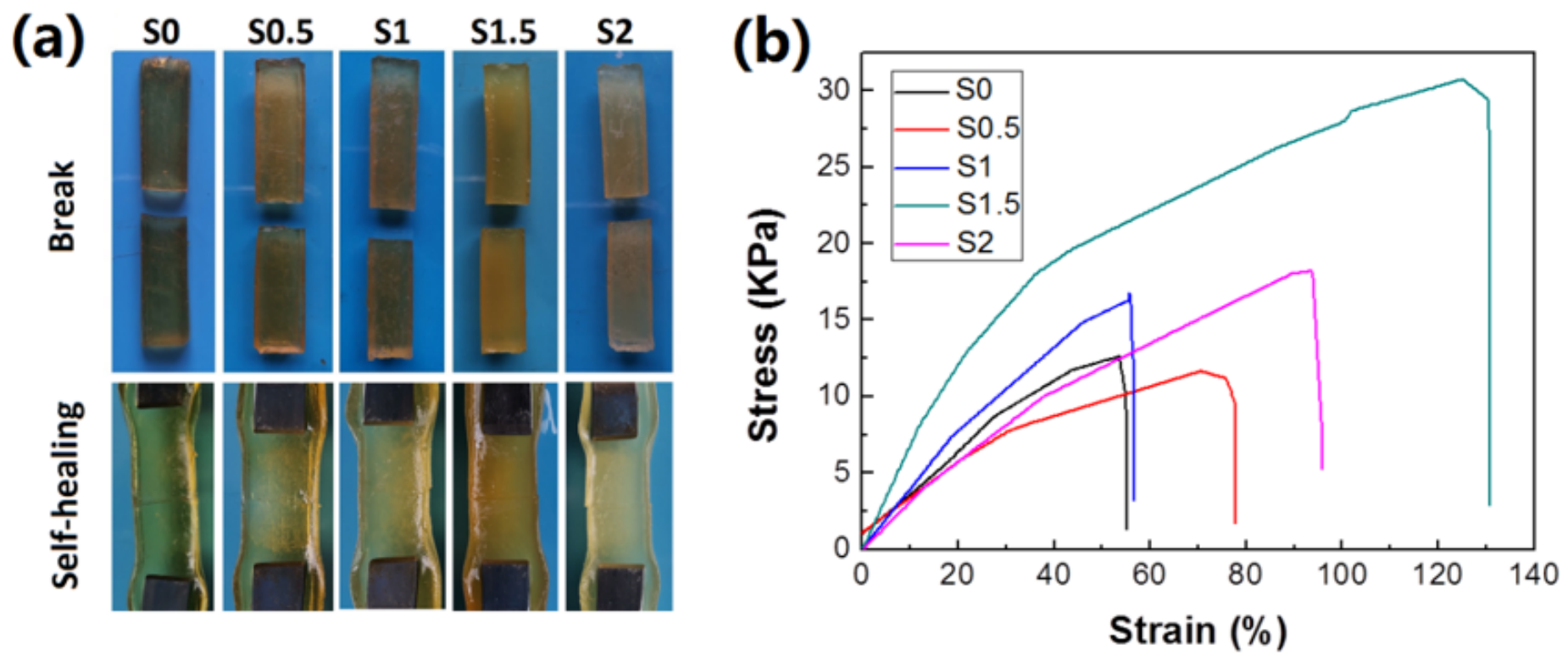

Figure 11

Self-healing properties of hydrogels. (a) Photograph of hydrogel self-healing before and after treatment. (b) Tensile curves of hydrogel after self-healing.
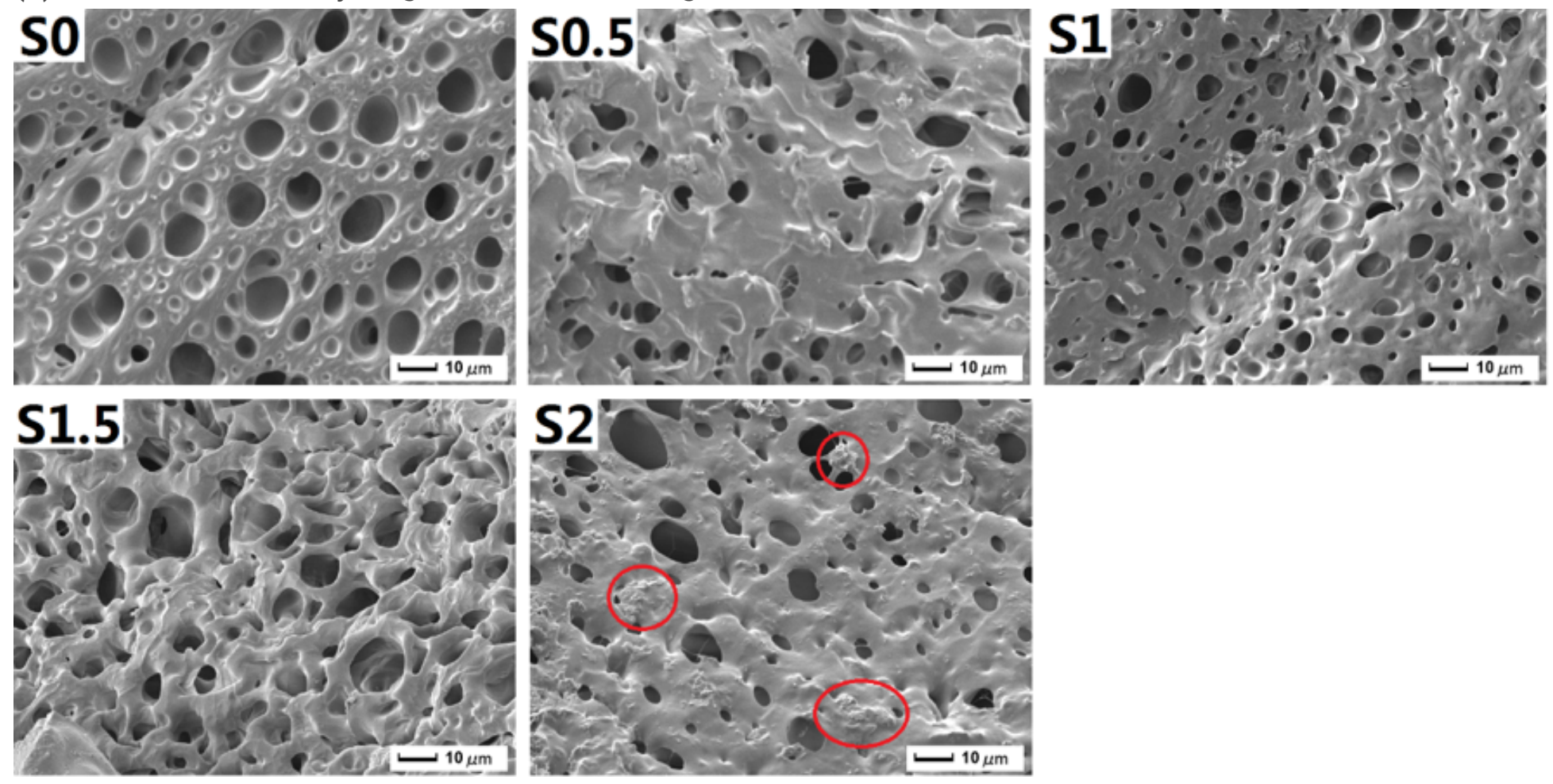

Figure 13

Morphology of fracture surface of TA/PAA hydrogels. The red circles in S2 mean aggregated JNSs. 